\title{
Type I autoimmune hepatitis: clinical course and outcome in an Italian multicentre study
}

\author{
A. FLOREANI*, G. NIRO†, E. ROSA RIZZOTTO*, S. ANTONIAZZI*, F. FERRARA*, I. CARDERI*, \\ V. BALDO\$, A. PREMOLI§, F. OLIVERO§, E. MORELLO§ \& M. DURAZZO§
}

\begin{abstract}
*Department of Surgical and Gastroenterological Sciences, University of Padova, Padova, Italy; †Department of Gastroenterology, Hospital Casa Sollievo della Sofferenza, San Giovanni Rotondo, Italy; †Department of Environmental Medicine and Public Health, Institute of Hygiene, University of Padova, Padova, Italy; §Department of Medicine, Ospedale Molinette, Torino, Italy

Correspondence to:

Dr A. Floreani, Department of Surgical and Gastroenterological Sciences, University of Padova, Via Giustiniani, 2, 35128 Padova, Italy. E-mail: annarosa.floreani@unipd.it
\end{abstract}

\section{Publication data}

Submitted 16 June 2006

First decision 16 July 2006

Resubmitted 25 July 2006

Accepted 28 July 2006

\section{SUMMARY}

\section{Background}

Many reports of autoimmune hepatitis (AIH) were written in the 'pre-Hepatitis C era' and data on the natural history are still incomplete.

\section{Aim}

To evaluate the clinical presentation and the natural history of type I $\mathrm{AIH}$.

\section{Methods}

Seventy-three consecutive patients with a regular follow-up of at least 2 years were prospectively included in the study. The mean follow-up was $91 \pm 61$ months.

\section{Results}

Patients with 'acute' onset at presentation were significantly older than patients with 'chronic' onset $(P<0.05)$ and had significantly higher serum levels of transaminase, $\gamma$-glutamyltransferase and bilirubin; Prothrombin time was significantly lower in the said group compared with AIH patients with 'chronic' onset. In 4 of 63 (6.3\%) female patients, AIH had the onset during pregnancy; in all of them the outcome of pregnancy was favourable. The major events during the follow-up included oesophageal varices $(n=9)$ and ascites $(n=$ 4), and 60 patients remained in remission while receiving immunosuppression. None of the patients died during the follow-up, but seven patients were transplanted. The cumulative transplant-free probability of survival was $73.5 \%$ at 280 months.

\section{Conclusions}

Elderly patients have more frequently an acute onset at presentation. Survival in AIH is apparently good; with early diagnosis, and improved medical therapy, liver transplantation for AIH will become a rare event in future. 


\section{INTRODUCTION}

Autoimmune hepatitis (AIH) is a chronic liver disease of unknown cause, which occurs in children and adults of all ages. ${ }^{1}$ In the presence of a histological picture of chronic hepatitis, diagnosis is based on characteristic clinical and biochemical findings, circulating autoantibodies and abnormal levels of serum globulins. ${ }^{1}$ It is a rare disease, with an estimated prevalence of approximately 170 cases per 1 million in northern Europe. ${ }^{2}$ On the basis of autoantibody pattern, AIH can be classified into three types: type I [characterized by antinuclear (ANA) and smooth muscle (SMA)antibodies]; type II [characterized by the presence of anti-liver-kidney microsomes type I (LKMI)] and type III [characterized by the positivity of soluble liver antigen (SLA)].

A scoring system proposed by the International Autoimmune Hepatitis Group (IAHG) to standardize the diagnosis for clinical trials provides support for diagnosing $\mathrm{AIH}^{3}$

The introduction in the late 1960s and 1970s of corticosteroid-based immunosuppressive regimens, with or without azathioprine as a steroid-sparing agent, dramatically improved the quality and length of life. During the subsequent years, the standard therapy for type I AIH with prednisolone or prednisone in monotherapy or in combination with azathioprine has been adopted worldwide. Remission of the disease is achieved on treatment in $65-80 \%$ of patients. ${ }^{4-6}$ One study considered the long-term follow-up of patients with maintenance therapy, with azathioprine, as being successful in approximately $80 \%$ of patients. ${ }^{7}$ A recent paper included 205 white North American patients with type I AIH evaluated between 1975 and 2005 at the Mayo Clinic. ${ }^{8}$ Roughly, 50\% of them have a follow-up of at least 5 years. The overall rate of remission during immunosuppression ranged from 50\% in younger to $61 \%$ in elderly patients.

A clinical trial from Canada in a large cohort of patients with AIH showed that patients who are asymptomatic at presentation have a good prognosis and may not require immunosuppressive therapy; cirrhosis on liver biopsy portends a poor prognosis in all patients. ${ }^{9}$ On the contrary, another study from the Mayo Clinic showed that histological cirrhosis does not diminish survival expectations. ${ }^{10}$ However, studies on the natural history of AIH in Europe are still lacking.
The aim of the present study was, therefore, to evaluate the clinical presentation and the natural history of AIH.

\section{MATERIALS AND METHODS}

\section{Patients}

The study considered all patients consecutively diagnosed with type I AIH between 1981 and 2004 in three Italian referring centres for AIH: University of Padova, Turin University and San Giovanni Rotondo Hospital. All patients had a regular follow-up of at least 2 years. Among them, 73 patients ( 63 females, 10 males, mean age $47.7 \pm 17.9$ years) were included.

The diagnosis of type I AIH was made by clinical, immunological and histological grounds. All patients had a positivity for ANA or antismooth muscle antibodies (ASMA); SLA was negative in all patients. All subjects were negative for HBV and HCV serum markers.

Acute presentation was defined by the presence of recent onset ( $<30$ days) symptoms (jaundice and/or fatigue and/or drowsiness) in conjunction with serum alanine transferase levels higher than 10-fold the upper normal limit). Most of these patients had been originally admitted to an 'infectious disease' ward with the diagnostic suspicion of acute viral hepatitis.

Patients with 'chronic' exhibited clinical and biochemical features compatible with chronic liver disorder. Symptoms had been persisting for at least 6 months and were usually minor and unspecific.

The revised scoring system for the diagnosis of AIH was calculated according to the report of the IAHG. ${ }^{3}$

\section{Evaluation of liver histology}

All patients included in the study underwent liver biopsy. Biopsy samples were examined in a blinded fashion using codes by a dedicated pathologist. The stains available included haematoxylin, stains for connective tissue, iron and copper-associated protein. The following histological features were recorded: portal inflammation, interface hepatitis (piecemeal necrosis), hepatocyte rosetting, ductular proliferation, bile duct damage, lobular necrosis, bridging necrosis and canalicular cholestasis. Liver biopsies of all individuals were graded and staged according to the internationally recognized histological scoring system. ${ }^{10}$ 


\section{Follow-up}

The patients were followed up regularly every 46 months by clinical and biochemical examination. Biochemical parameters included AST, ALT, alkaline phosphatase, immunoglobulins, bilirubin, red blood cell, white blood cell and platelet counts, prothrombin time and $\alpha$-foetoprotein.

Liver ultrasound was performed every 12 months in precirrhotic patients and every 6 months in patients with cirrhosis. The mean follow-up was $91 \pm 61$ months.

\section{Treatment}

All patients (with either 'acute' or 'chronic' onset) were treated with prednisolone or prednisone (initial dosage of $0.5 \mathrm{mg} / \mathrm{kg} / \mathrm{day}$ ) associated with steroid-sparing azathioprine (initial dosage of $2 \mathrm{mg} / \mathrm{kg} / \mathrm{day}$ ) with a tailored maintenance dosage of $10 \mathrm{mg} /$ day of steroid and $50-75 \mathrm{mg} /$ day of azathioprine. Only one patient experienced intolerance to azathioprine and received prednisolone as monotherapy. Compliance with the treatment and side effects were assessed by means of personal interviews every 6 months.

Bone mineral density was assessed by dual-photon $\mathrm{X}$-ray absorptiometry at the lumbar spine every 2 years. Patients with osteoporosis received i.m. disodium clodronate $100 \mathrm{mg}$ every 10 days and calcium plus vitamin D supplements.

Withdrawal of immunosuppressive therapy after 2 years on attainment of normal level of enzymes was achieved in only one patient (a 69-year-old male), but he also experienced a relapse within 6 months, and immunosuppression was started again.

\section{Laboratory methods}

Autoantibodies (ASMA, ANA, AMA and LKM) were tested by indirect immunofluorescence method using normal human gastric mucosa and normal rat kidney and liver. Sera were tested at an initial dilution of 1:5 and were considered to be positive when they produced a reaction at a dilution of $\geq 1: 40$. Antineutrophil cytoplasmic antibodies (ANCA) were determined by indirect immunofluorescence. The patient's serum (diluted 1:20, 1:40) was applied to neutrophils previously fixed in ethanol and incubated with F(ab)2 fragment of rabbit anti-human immunoglobulin G chain labelled with fluorescein isothiocyanate (Alifax Diag- nostici, Padova, Italy). Immunofluorescent staining in the perinuclear zone (p-ANCA) or cytoplasmic compartment (c-ANCA) in the 1:20 sample was taken as positive.

Hepatitis B (HBsAg and anti-HBc) infection was determined by ELISA using commercially available kits (Abbott Laboratories, North Chicago, IL, USA).

Testing for anti-HCV was performed in duplicate by second and third generation ELISA (Ortho Diagnostic system, Raritan, NJ, USA) and confirmed by HCV-RNA detection using a commercially available qualitative polymerase chain reaction assay (Amplicor Monitor assay; Roche Diagnostic System, Branchburg, NJ, USA). HCV testing in patients enrolled before 1990 was performed on stored sera during follow-up.

The other biochemical variables were assayed by standard methods.

\section{Statistical analysis}

Quantitative data are expressed as median and range. The $t$-test was used for quantitative data, Chi-squared test for qualitative data and Wilcoxon test for matched pairs. Survival was analysed according to the KaplanMeier method and was assessed with the log-rank test. Statistical analysis was performed using SPSS software (SPSS, Chicago, IL, USA).

\section{RESULTS}

The clinical details of patients at presentation are summarized in Table 1 (data are expressed as mean \pm s.d.). Sixty-three patients were females. The mean age was $48.8 \pm 17.9$ years. At presentation, a definite IAHG pretherapy score was fulfilled by 48 patients (65.8\%); the remaining 24 patients had a probable diagnosis of AIH (32.99\%). Twenty-eight subjects (38.4\%) had cirrhosis at presentation.

Patients with acute onset at presentation $(n=27)$ were significantly older than patients with chronic onset $\quad(n=46) \quad(56 \pm 20 \quad$ vs. $45.5 \pm 15$ years, $P<0.05)$. Moreover, they exhibited significantly higher levels of serum transaminases, GGT, total bilirubin $(P<0.001)$ than patients with chronic onset. They also exhibited significantly lower mean prothrombin percentage $(P<0.001)$ and serum albumin $(P<0.05)$ compared with the latter group (Table 2$)$. AIH scores or antibody profiles were not different between the two groups. 
Table 1. Clinical details of patients at presentation (mean \pm s.d.)

No. of patients

Age

Female (\%)

AST (U/L)

ALT (U/L)

GGT (U/L)

$\operatorname{ALP}(\mathrm{U} / \mathrm{L})$

$\operatorname{IgG}(\mathrm{mg} / \mathrm{dL})$

$\operatorname{IgA}(\mathrm{mg} / \mathrm{dL})$

$\operatorname{IgM}(\mathrm{mg} / \mathrm{dL})$

PT (\%)

Serum albumin $(\mathrm{g} / \mathrm{L})$

Total bilirubin (mg/dL)

Conjugated bilirubin $(\mathrm{mg} / \mathrm{dL})$

ANA $(\geq 1: 40)(\%)$

SMA $(\geq 1: 40)(\%)$

ANA + SMA $(\geq 1: 40)(\%)$

Probable IAHG pretherapy score (\%)

Definite IAHG pretherapy score (\%)

ALT, alanine amino transferase; AST, aspartate amino transferase; GGT, $\gamma$-glutamyl transferase; ALP, alkaline phosphatase; IgG, immunoglobulin G; IgA, immunoglobulin A; IgM, immunoglobulin M; PT, prothrombin time.

In the medical history of patients with acute onset, we could identify the assumption of a drug that appears as a 'trigger agent' for AIH in five subjects (four of them were older than 65 years). The recorded medications were trazodone in one, nimesulide in three and thorvastatin in one.

Overall, 16 patients experienced the onset of AIH in their geriatric life (>65 years) and 57 in adult life (Table 3). Patients over 65 years had significantly higher levels of both AST $(P<0.02)$ and GGT $(P<0.01)$ than patients below 65 years of age. The mean histological grade before treatment was similar in the two groups of patients, while the histological stage, an indicator of hepatic fibrosis, was significantly higher in older patients compared with the patients below 65 years of age $(P<0.003)$. The number of patients with cirrhosis (i.e. stage 6 at presentation) was $10(62.5 \%)$ in the $>65$ group and $18(31.5 \%)$ in the $<65$ group $(P<0.001)$ (Table 3$)$.

As expected, 31 of 73 patients with type I AIH (42.5\%) had association with extrahepatic autoimmune conditions. The most frequent condition was autoimmune thyroid dysfunction (23.3\%) (Table 4).
Table 2. Clinical features of patients with acute onset and chronic onset AIH (mean \pm s.d.)

\begin{tabular}{lccc}
\hline & Acute onset & Chronic onset & $P$-value \\
\hline No. of patients & 27 & 46 & - \\
Age (mean \pm s.d.) & $56.0 \pm 20.0$ & $45.5 \pm 15.0$ & $<0.05$ \\
Age $>65$ years $(n)$ & $11(40 \%)$ & $5(11 \%)$ & $<0.005$ \\
Female (\%) & 88.9 & 84.8 & - \\
AST (U/L) & $796.2 \pm 528.4$ & $299.7 \pm 326.4$ & $<0.001$ \\
ALT (U/L) & $901.0 \pm 579.7$ & $368.5 \pm 405.4$ & $<0.001$ \\
GGT (U/L) & $203.9 \pm 138.9$ & $109.4 \pm 74.1$ & $<0.001$ \\
ALP (U/L) & $266.7 \pm 124.9$ & $228.4 \pm 177.9$ & N.S. \\
IgG (mg/dL) & $2386.8 \pm 685.8$ & $2298.7 \pm 1077.1$ & N.S. \\
IgA (mg/dL) & $335.3 \pm 180.5$ & $309.9 \pm 202.8$ & N.S. \\
IgM (mg/dL) & $216.3 \pm 160.2$ & $152.8 \pm 80.8$ & 0.04 \\
PT (\%) & $65.2 \pm 16.5$ & $76.7 \pm 16.6$ & $<0.001$ \\
Serum albumin & $33.3 \pm 5.2$ & $30.6 \pm 15.3$ & $<0.05$ \\
$\quad$ (g/L) & & & \\
Total bilirubin & $5.4 \pm 6$ & $1.4 \pm 0.9$ & $<0.001$ \\
(mg/dL) & & & \\
Conjugated & $3.8 \pm 4.4$ & $0.7 \pm 0.7$ & $<0.001$ \\
bilirubin (mg/dL) & & &
\end{tabular}

ALT, alanine amino transferase; AST, aspartate amino transferase; GGT, $\gamma$-glutamyl transferase; ALP, alkaline phosphatase; IgG, immunoglobulin G; IgA, immunoglobulin A; IgM, immunoglobulin M; PT, prothrombin time.

Table 5 summarizes the relationship between $\mathrm{AIH}$ and pregnancy. Of 63 females (6.3\%), four had onset of the disease during pregnancy, and the outcome of pregnancy in these patients was favourable. The total number of pregnancies was 94 (93.6\%) before the onset of the disease and 6 (6.4\%) after the diagnosis of $\mathrm{AIH}$, during the pharmacological remission. During pregnancy, immunosuppression was continued, but the maintenance dosage was very low $(5-7.5 \mathrm{mg} /$ day of prednisolone plus $50 \mathrm{mg} /$ day of azathioprine). Among these, two patients experienced miscarriage and the remaining had a normal vaginal delivery. Overall no alteration of the fertile life was noted. Particularly, the mean age of menarche was $12.8 \pm 1.7$ years and the mean age of menopause was $50.5 \pm 3.8$ years.

\section{Follow-up}

The mean follow-up was $91 \pm 61$ months.

The major events in the group with $\mathrm{AIH}$ included oesophageal varices $(n=9)$ and ascites $(n=4)$, and 60 patients remained in remission while receiving immunosuppression (Table 6). 
Table 3. Clinical features of patients with type I AIH with onset in geriatric age ( $>65$ years) and in adult life (<65 years) (mean \pm s.d.)

\begin{tabular}{|c|c|c|c|}
\hline & $<65$ years & $>65$ years & $P$-value \\
\hline No. of patients & 57 & 16 & - \\
\hline $\begin{array}{l}\text { No. of patients } \\
\text { with acute } \\
\text { onset }\end{array}$ & $16(28 \%)$ & $11(68.7 \%)$ & $<0.05$ \\
\hline Age (mean \pm s.d.) & $42.0 \pm 14.0$ & $73.0 \pm 5.0$ & - \\
\hline Female $(\%)$ & 89.9 & 86.7 & - \\
\hline \multicolumn{4}{|l|}{$\begin{array}{l}\text { Histological } \\
\text { findings* at } \\
\text { presentation }\end{array}$} \\
\hline Mean grade & $5.83 \pm 0.96$ & $5.41 \pm 0.61$ & N.S. \\
\hline Mean stage & $4.62 \pm 1.08$ & $5.47 \pm 0.62$ & $<0.003$ \\
\hline AST (U/L) & $426.5 \pm 454.6$ & $629.1 \pm 497.0$ & N.S. \\
\hline ALT (U/L) & $513.3 \pm 499.8$ & $689.8 \pm 640.1$ & N.S. \\
\hline GGT (U/L) & $121.9 \pm 103.2$ & $214.7 \pm 105.1$ & $<0.01$ \\
\hline ALP (U/L) & $235.2 \pm 162.7$ & $264.9 \pm 162.1$ & N.S. \\
\hline IgG (mg/dL) & $2239.9 \pm 984.8$ & $2287.1 \pm 875.7$ & N.S. \\
\hline IgA (mg/dL) & $306.7 \pm 173.8$ & $362.0 \pm 256.5$ & N.S. \\
\hline IgM (mg/dL) & $175.8 \pm 121.9$ & $173.1 \pm 107.3$ & N.S. \\
\hline PT (\%) & $73.6 \pm 17.6$ & $69.5 \pm 16.8$ & N.S. \\
\hline $\begin{array}{l}\text { Serum } \\
\text { albumin (g/L) }\end{array}$ & $36.5 \pm 5.2$ & $34.3 \pm 7.5$ & N.S. \\
\hline $\begin{array}{l}\text { Total } \\
\text { bilirubin }(\mathrm{mg} / \mathrm{dL})\end{array}$ & $2.8 \pm 4.4$ & $2.7 \pm 2.5$ & N.S. \\
\hline $\begin{array}{l}\text { Conjugated } \\
\text { bilirubin }(\mathrm{mg} / \mathrm{dL})\end{array}$ & $1.8 \pm 3.2$ & $1.8 \pm 2.1$ & N.S. \\
\hline
\end{tabular}

* Maximum grade, 18; maximum stage, 6.
Table 4. Associated autoimmune conditions

Autoimmune thyroid dysfunction (\%)

Type I diabetes (\%)

Sjogren's syndrome (\%)

Antiphospholipid syndrome (\%)

Vitiligo (\%)

Cryoglobulinaemia (\%)

Autoimmune polyendocrine syndrome type III (\%)

Coeliac disease $(\%)$

Total $(\%)$
$17(23.3)$

$5(6.8)$

$3(4.1)$

$1(1.3)$

$1(1.3)$

$1(1.3)$

$1(1.3)$

$1(1.3)$

$30(41)$
During follow-up, seven patients received orthotopic liver transplantation (OLTx) (9.5\%), two in the group with cirrhosis at presentation and five in the group without cirrhosis. Indications for OLTx included hepatocellular carcinoma (HCC) (in one patient and complications of portal hypertension in the remaining patients). The mean time from the diagnosis of AIH to
Table 5. Obstetric and gynaecological features in patients with AIH

63 women

No. of females with onset during pregnancy

$4(6.3 \%)$

No. of pregnancies before the onset

$88 / 94(93.6)$

No. of pregnancies postdiagnosis of $\mathrm{AIH}$

$6 / 94(6.4 \%)$

Miscarriages preceding the diagnosis

$11 / 13(74.6 \%)$

Miscarriages postdiagnosis

Age of menarche (mean \pm s.d.)

Age of menopause (mean \pm s.d.)

$2 / 13(15.4 \%)$

No. of females with

abnormalities of menstrual cycle

$12.8+1.6$

$49.7 \pm 4.1$

$13(21.3 \%)$

Table 6. Outcome of patients according to the presence or absence of cirrhosis at presentation

$\begin{array}{lll}\text { Cirrhosis at } & \text { No cirrhosis } & \\ \text { presentation } & \text { at presentation } & \text { All patients } \\ (n=28)(\%) & (n=45)(\%) & (n=73)(\%)\end{array}$

\begin{tabular}{llll}
\hline $\begin{array}{l}\text { End points } \\
\text { Transplant }\end{array}$ & $2(7.1)$ & $5(11.1)$ & $7(9.5)$ \\
Oesophageal & $4(14.3)$ & $5(11.1)$ & $9(12.3)$ \\
$\quad$ varices & & & \\
Ascites & $2(7.1)$ & $2(4.4)$ & $4(5.4)$ \\
HCC & - & $1(2.2)$ & $1(1.4)$
\end{tabular}

transplant was $71.1 \pm 48.4$ months. Figure 1 shows the estimated probability of transplant in our population (considering only 57 subjects $<65$ years of age). The cumulative transplant-free probability of survival was $73.5 \%$ at 280 months.

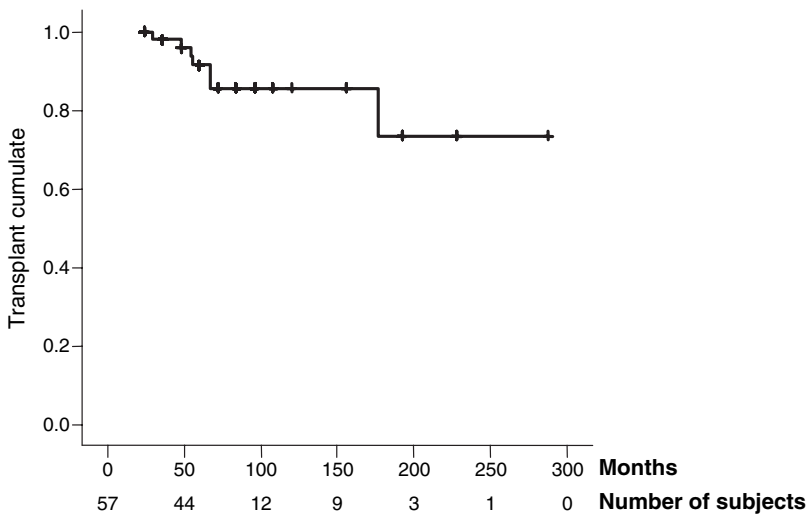

Figure 1. Kaplan-Meier estimation for transplant curve according to the disease in subjects $<65$ years of age. 


\section{DISCUSSION}

This multicentre Italian study is representative of a relatively large series of type I AIH hepatitis patients with a long-term follow-up. In general, the clinical presentation of AIH in Italy is not different from other series. Some clinical details, however, should be stressed. First, $21.7 \%$ of patients with type I AIH are older than 65 years at presentation. Moreover, patients with acute onset have a significantly higher age than those with chronic onset. This is in agreement with other reports in which $17-56 \%$ cases of all patients are over 65 years old at presentation. ${ }^{8,11,12,13}$ These subjects most commonly presented with signs of acute icteric hepatitis. Moreover, elderly patients have a significantly higher frequency of cirrhosis at presentation than adults, but the prognosis is excellent: very few elderly patients with AIH have clinically aggressive disease (overt jaundice, ascites and encephalopathy). AIH should be considered in the older patient, nonetheless, to avoid any delay in starting immunosuppressive therapy. The higher staging score found in elderly patients compared with adults is in agreement with the recent report by Czaja et al., ${ }^{8}$ who observed a greater frequency of cirrhosis at presentation in elderly patients compared with the group $<30$ years of age.

GGT serum levels were found significantly higher in subjects $>65$ compared with $<65$ years, whereas no significant difference in ALP was found between the two groups. In other words, the increase in GGT does not follow ALP. This finding is difficult to explain. A possible explanation for the increase in GGT, at least in part, could be the enzymatic induction in the patients with the history of drug assumption before the onset of AIH.

In fact, another point is the role of drugs as potential triggers. Certain drugs are known as triggers of $\mathrm{AIH}^{1}$; however, nimesulide and trazodone have not been associated with AIH previously. Nimesulide toxicity has been widely associated with adverse reactions of the liver, including increases in serum aminotransferase activities, hepatocellular necrosis and/or intrahepatic cholestasis. ${ }^{14}$ Drug-induced liver toxicity from trazodone has also been reported, ${ }^{15}$ but data on the follow-up of patients are lacking. It is notable, however, that the patient who developed AIH after trazodone therapy experienced a severe acute onset of AIH. Autoantibodies were negative at that time, but both ANA and SMA became positive after 4 weeks from the acute onset, and titre increased to $1: 320$ and 1:1280, respectively, within 3 months. Similar to other drugs causing hepatotoxicity, both the molecule and the patient contribute to the hazard. Genetic susceptibility of the patient may be one of the factors that trigger autoimmunity. The list of drugs considered as potential triggers for $\mathrm{AIH}$, however, can be longer, and it is advisable to notify all adverse reactions of drugs to the National Register.

We put particular emphasis on recording data concerning menstrual abnormalities and the relationship between pregnancy and AIH. We failed to observe alterations in menstrual abnormalities and menopause in our patients. Four females experienced the onset of the disease during pregnancy, and the outcome in such patients was favourable. The total number of pregnancies was 93, six after achieving the remission. The miscarriage rate of $15.4 \%$ is in agreement with the previously reported data in the largest case series. ${ }^{16,17}$ As previously reported, ${ }^{16}$ successful completion of pregnancy is realistic expectation for patients with well controlled AIH. A careful monitoring of pregnancy is, however, mandatory. No abnormalities in the foetus were observed. We also failed to observe severe flare-ups after delivery. ${ }^{18,} 19$

This study confirms that remission can be induced and maintained in most AIH patients on the standard corticosteroid-based immunosuppression used for several decades. This is despite nearly $38.4 \%$ of our cohort being cirrhotic at presentation, which is high compared with $14 \%$ in another recent series of 89 patients, ${ }^{10}$ but is consistent with other data showing that the presence of cirrhosis does not affect steroid responsiveness. ${ }^{20}$ However, 7 of the 73 type I AIH patients in our series required liver transplantation for end-stage liver disease, two of whom were cirrhotic at presentation and five did not present cirrhosis at the initial biopsy. Another recent large series of 103 patients from a single centre in Germany found that fewer patients (29\%) had histological evidence of cirrhosis at presentation and no OLT was performed after a mean observation period of 95 months. ${ }^{21}$ The overall transplant rate during the follow-up is similar to that observed by Feld et al. in Canada. ${ }^{9}$ These authors, however, reported a significantly higher transplant rate in subjects with cirrhosis (14.2\%) compared with the group without cirrhosis at presentation (1.2\%). In our experience, deterioration of liver disease is because of the failure of immunosuppressive therapy, despite 
the presence of cirrhosis at initial biopsy. In a large context, examining this issue, Roberts et al. ${ }^{22}$ came to the same conclusion.

No deaths were recorded during follow-up. One patient developed HCC and received liver transplant. The cumulative transplant-free probability of survival was $73.5 \%$ at 280 months, which is a good result, considering different types of liver disease.
In conclusion, with earlier diagnosis and improved medical therapy, liver transplantation for AIH will become a rare event in the future.

\section{ACKNOWLEDGEMENTS}

This study was funded by grant of the MURST National Research Programme.

\section{REFERENCES}

1 Krawitt EL. Autoimmune hepatitis. N Engl J Med 2006; 354: 54-66.

2 Boberg KM, Aadland E, Jahnsen J, Raknerud N, Stiris M, Bell H. Incidence and prevalence of primary biliary cirrhosis, primary sclerosing cholangitis and autoimmune hepatitis in a Norwegian population. Scand J Gastroenterol 1998; 33: 99-103.

3 Alvarez F, Berg PA, Bianchi FB, et al. International autoimmune hepatitis group report: review of criteria for diagnosis of autoimmune hepatitis. $J$ Hepatol 1999; 31: 929-38.

4 Czaja AJ. Drug therapy in the management of type 1 autoimmune hepatitis. Drugs 1999; 57: 49-68.

5 Heneghan MA, McFarlane IG. Current and novel immunosuppressive therapy for autoimmune hepatitis. Hepatology 2002; 357: 7-13.

6 Czaja AJ, Bianchi FB, Carpenter HA, et al. Treatment challenges and investigational opportunities in autoimmune hepatitis. Hepatology 2005; 41: 207-15.

7 Johnson P, McFarlane IG, Williams R. Azathioprine for long-term maintenance of remission in autoimmune hepatitis. $N$ Engl J Med 1995; 333: 958-63.

8 Czaja AJ, Carpenter HA. Distinctive clinical phenotype and treatment outcome of type 1 autoimmune hepatitis in the elderly. Hepatology 2006; 43: 532-8.

9 Feld JJ, Dinh H, Arenovich T, Marcus VA, Wanless IR, Heathcote EJ. Autoimmune hepatitis: effect of symptoms and cirrhosis on natural history and outcome. Hepatology 2005; 42: 53-62.

10 Ishak K, Baptista A, Bianchi L, et al. Histological grading and staging of chronic hepatitis. J Hepatol 1995; 22: 696-9.

11 Newton JL, Burt AD, Park JB, Matthew $\mathrm{J}$, Bassendine MF, James OFW. Autoimmune hepatitis in older patients. Age Ageing 1997; 26: 441-4.

12 Parker DR, Kingham JGC. Type I autoimmune hepatitis is primarily a disease of later life. Q J Med 1997; 90: 289-96.

13 Schramm C, Kanzler S, zum Buschenfelde KH, Galle PR, Lohse AW. Autoimmune hepatitis in the elderly. Am J Gastroenterol 2001; 96: 1587-91.

14 Boelsterli UA. Mechanisms of NSAIDinduced hepatotoxicity: focus on nimesulide. Drug Saf 2002; 25: 633-48.

15 Conforti A, Leone R, Ghiotto E, et al. Spontaneous reporting of drug-related hepatic reactions from two Italian regions (Lombardy and Veneto). Dig Liver Dis 2000; 32: 16-23.

16 Heneghan MA, Norris SM, O'Grady JG, Harrison PM, McFarlane IG. Manage- ment and outcome of pregnancy in autoimmune hepatitis. Gut 2001; 48: 97-102.

17 Schramm C, Herkel J, Beuers U, Kanzler S, Galle PR, Lohse AW. Pregnancy in autoimmune hepatitis: outcome and risk factors. Am J Gastroenterol 2006; 101: 556-60.

18 Buchel E, Van Steenbergen W, Nevens F, Fevery J. Improvement of autoimmune hepatitis during pregnancy followed by flare-up after delivery. Am $J$ Gastroenterol 2002; 97: 3160-5.

19 Samuel D, Riordan S, Strasser S, Kurtovic J, Singh-Grewel I, Koorey D. Severe autoimmune hepatitis first presenting in early post partum period. Clin Gastroenterol Hepatol 2004; 2: 622-4.

20 Omagari K, Kinoshita $\mathrm{H}$, Kato $\mathrm{Y}$, et al. Clinical features of 89 patients with autoimmune hepatitis in Nagasaki Prefecture, Japan. J Gastroenterol 1999; 34: 221-6.

21 Kanzler S, Lohr H, Gerken G, Galle PR, Lohse AW. Long-term management and prognosis of autoimmune hepatitis (AIH): a single center experience. $Z$ Gastroenterol 2001; 39: 339-41.

22 Roberts SK, Therneau TM, Czaja AJ. Prognosis of histological cirrhosis in type I autoimmune hepatitis. Gastroenterology 1996; 110: 848-57. 